\title{
Effect of initial conditions on the speed of reaction-diffusion fronts
}

\author{
Vicenç Méndez, ${ }^{1}$ Joaquim Fort, ${ }^{2}$ and Toni Pujol ${ }^{2}$ \\ ${ }^{1}$ Departament de Medicina, Facultat de Ciències de la Salut, Universitat Internacional de Catalunya, c/ Gomera s/n, \\ 08190-Sant Cugat del Vallès (Barcelona), Spain \\ ${ }^{2}$ Departament de Física, Universitat de Girona, Campus Montilivi, 17071 Girona, Catalonia, Spain
}

(Received 7 October 2002; published 28 January 2003)

\begin{abstract}
The effect of initial conditions on the speed of propagating fronts in reaction-diffusion equations is examined in the framework of the Hamilton-Jacobi theory. We study the transition between quenched and nonquenched fronts both analytically and numerically for parabolic and hyperbolic reaction diffusion. Nonhomogeneous media are also analyzed and the effect of algebraic initial conditions is also discussed.
\end{abstract}

DOI: 10.1103/PhysRevE.67.016213

PACS number(s): 05.45.-a, 87.10.+e, 05.40.Jc, 05.60.Cd

\section{INTRODUCTION}

Reaction-diffusion equations admit wave-front solutions propagating with their minimum possible speed, provided that they start from an initial profile with compact support (compact means different from zero in a finite region). For a parabolic reaction-diffusion (PRD) equation with logistic reactive kinetics, Fisher derived a lower bound for the speed of wave fronts in 1937 [1]. That this bound coincides with their exact speed, was proved by Kolmogorov, Petrovskii, and Piskunov (KPP) [2] also in 1937. Aronson and Weinberger showed that the minimal-speed wave is also selected, as long as the initial condition has compact support, for a large class of reactive kinetics, in several dimensions, and also for discrete-time and dispersive-kernel models [3-6].

Noncompact initial conditions are also important, but they are often ignored in wavefront speed research (for a recent review see Ref. [7]). Noncompact initial conditions have applications in physics and biology (see, e.g., Ref. [8]), because, in general, the profile observed initially may well be closer to a non-compact (e.g., exponential) function than to a compact (e.g., step) one. Mollison [9] showed that from the initial profile $e^{-\alpha x}$ as $x \rightarrow \infty$, fronts emerge traveling with the minimum speed ( $v=2$ for PRD equations) if $\alpha$ is higher than a critical value $\alpha^{*}$ ( $\alpha^{*}=1$ for PRD equations), that is, if the initial condition is sufficiently steep. When $\alpha<\alpha^{*}$ wave fronts also exist but they travel with a speed that depends explicitly on the initial condition through $\alpha$. This kind of fronts are often referred to as quenched or degenerated fronts. Mollison developed a method for finding the relation between the speed and the initial condition consisting in linearizing the PRD-logistic (i.e., Fisher) equation around the unstable state (thus, effectively considering Kendall's equation) and assuming the profile $e^{-\alpha(x-v t)}$ as $x \rightarrow \infty$ [9]. This method holds only if one takes $e^{-\alpha x}$ as initial condition. For the PRD logistic (i.e., Fisher) equation, some exact (Ref. [10] and Sec. 11.4 in Ref. [11]) and approximate (Ref. [12] and Sec. 11.3 in Ref. [11]) solutions have been found. They correspond to very specific initial conditions. PRD equations (both logistic and nonlogistic) have been considered in Ref. [13], where accelerating fronts have been found for initial data with sufficiently slow decay.

In this work we will consider compact versus noncompact initial conditions, including more general systems than those described by PRD equations. We use a WKB transformation which in the limit $x \rightarrow \infty$ and $t \rightarrow \infty$ converts the reactiondiffusion equation, with KPP kinetics, into a HamiltonJacobi equation. Making use of the known solutions for the Hamilton-Jacobi equation in the framework of the classical mechanics one can get the position of the front for large times. This allows us to calculate the asymptotic speed of the front [14]. Following the ideas developed by Freidlin $[15,16]$, we extend the Hamilton-Jacobi formalism in order to incorporate the effect of the initial condition, exponential or not, and in consequence we will generalize the results by Aronson and Weinberger. For instance, we study the transition from quenched to nonquenched fronts for hyperbolic reaction-diffusion (HRD) equations starting from an exponential initial condition. Moreover, we also show that nonquenched fronts may also be obtained from initial conditions decaying slower than $e^{-x}$, for example, from algebraic initial conditions. We also study how the exponential initial condition affects the speed of fronts in heterogenous-media PRD equations, where again Mollison's method does not hold.

\section{THEORETICAL FORMULATION}

In order to study the implications of the initial conditions on the speed of fronts, we convert the reaction-diffusion problem into a Hamilton-Jacobi equation, which has a wellknown general solution. In order to do so, we follow the method for large deviations developed by Freidlin $[15,16]$.

We consider the general problem

$$
\begin{gathered}
\partial_{t} \rho(x, t)=\mathcal{F}\left(\partial_{t t} \rho, \partial_{x} \rho, \partial_{x x} \rho, \ldots\right)+f(\rho), \\
\rho(x, 0)=g(x),
\end{gathered}
$$

where the reactive kinetics term, $f(\rho)$, is such that $f(0)$ $=f(1)=0, f(\rho)>0$ for $\rho \in(0,1), f(\rho)<0$ for $\rho \notin[0,1]$ and $f^{\prime}(0)=\max _{0 \leqslant \rho \leqslant 1} f^{\prime}(\rho)$. Then we assume that $f(\rho)$ has an unstable state $(\rho=0)$, and a stable one $(\rho=1)$. The class of such reaction functions is called KPP reaction terms. First of all, let us consider a step function as initial condition, that is,

$$
g(x)=\theta(-x)= \begin{cases}1, & x \leqslant 0 \\ 0, & x>0\end{cases}
$$


The hyperbolic scaling procedure $x \rightarrow x / \varepsilon, t \rightarrow t / \varepsilon$ allows us to work in the large deviation limit, that is, in the large-scale and large-time limit when $\varepsilon \rightarrow 0$. Thus the function $\rho^{\varepsilon}(x, t)$ $=\rho(x / \varepsilon, t / \varepsilon)$ is a solution to the problem

$$
\begin{gathered}
\varepsilon \partial_{t} \rho^{\varepsilon}(x, t)=\mathcal{F}\left(\varepsilon^{2} \partial_{t t} \rho^{\varepsilon}, \varepsilon \partial_{x} \rho^{\varepsilon}, \varepsilon^{2} \partial_{x x} \rho^{\varepsilon}, \ldots\right)+f\left(\rho^{\varepsilon}\right), \\
\rho^{\varepsilon}(x, 0)=\theta(-x) .
\end{gathered}
$$

As the system has two equilibrium states, one being stable and the other one unstable, it is assumed that from initial conditions the traveling wave $\rho(x, t)=\psi(x-v t)$ connects both states by invading the unstable one for large $t(\varepsilon \rightarrow 0)$. Thus $\lim _{\varepsilon \rightarrow 0} \rho^{\varepsilon}(x, t)=\psi[x-v t / \varepsilon]$ and $\rho^{\varepsilon}(x, t) \rightarrow 0$ if $x>v t$ and $\rho^{\varepsilon}(x, t) \rightarrow 1$ if $x<v t$. Therefore, the solution of Eq. (3) tends to the step function $\theta(v t-x)$ when $\varepsilon \rightarrow 0$, where $v$ is the speed of the front. The zero approximation in Eq. (3) for $\varepsilon \rightarrow 0$ is characterized by the speed only, and not by the shape of the front which should appear for higher orders.

The problem then is to derive the evolution equation for the reaction front separating the region where $\rho^{\varepsilon}=0$ and the region where $\rho^{\varepsilon}=1$. Since $\rho^{\varepsilon}(x, t) \geqslant 0$ we can make the WKB transformation

$$
\rho^{\varepsilon}(x, t)=e^{-G^{\varepsilon}(x, t) / \varepsilon}, \quad G^{\varepsilon}(x, t) \geqslant 0,
$$

where $G^{\varepsilon}(x, t)$, which will determine the location of the front in the limit $\varepsilon \rightarrow 0$, satisfies

$$
\partial_{t} G^{\varepsilon}+\mathcal{F}\left(\partial_{t} G^{\varepsilon}, \partial_{t t} G^{\varepsilon}, \partial_{x} G^{\varepsilon}, \partial_{x x} G^{\varepsilon}, \ldots\right)+f\left(\rho^{\varepsilon}\right) / \rho^{\varepsilon}=0 .
$$

Since $e^{-G^{\varepsilon}(x, t) / \varepsilon} \rightarrow 0$ as $\varepsilon \rightarrow 0$ for $G^{\varepsilon} \geqslant 0$, the function

$$
G(x, t)=\lim _{\varepsilon \rightarrow 0} G^{\varepsilon}(x, t) \geqslant 0
$$

obeys

$$
\partial_{t} G+H\left(\partial_{x} G, \ldots\right)=0,
$$

which is the classical Hamilton-Jacobi equation corresponding to the reaction-diffusion problem. Analogously to classical mechanics, here $H=-\partial_{t} G>0$ is the Hamiltonian function, $p=\partial_{x} G$ is the conjugated momenta, and $G(x, t)$ plays the role of the action and, therefore, the solution of Eq. (5) is given by the variational problem

$$
G(x, t)=\min _{y}\left\{\int_{0}^{t} L[x(s), p(s)] d s, x(t)=x, x(0)=y \in S_{0}\right\},
$$

where $S_{0}$ is the support of the initial condition and $L$ is the Lagrangian function, as in classical mechanics, $L=\dot{x}(s) p$ $-H$ and $\dot{x}(s)=d x(s) / d s$. Once one gets $G(x, t)$, the location of the front at time $t$ will be given by the equation $G(x, t)=0$. Note from Eq. (6) that $G(x, 0)=0$ for $g(x)=\theta$ $(-x)$.

If the initial condition has no compact support or it is not a steplike function, then some generalization to Eq. (6) must be done [15]. For example, let now the initial condition be

$$
g(x)= \begin{cases}1, & x<0 \\ e^{-h(x)}, & x>0,\end{cases}
$$

where $h(x)$ is a positive function increasing with $x$. In this case $G^{\varepsilon}(x, 0)=\varepsilon h(x / \varepsilon)$ for $x>0$ and $G^{\varepsilon}(x, 0)=0$ for $x<0$. The simplest way to generalize Eq. (6) in order to incorporate the effect of the initial condition is by adding $G(x, 0)$ and therefore one has [15]

$$
\begin{aligned}
& G(x, t)= \min _{y \geqslant 0}\{\varepsilon h(y / \varepsilon) \\
&\left.+\int_{0}^{t} L[x(s), p(s)] d s, x(t)=x, x(0)=y\right\}, \\
& G(x, t)=\min _{y \leqslant 0}\left\{\int_{0}^{t} L[x(s), p(s)] d s, x(t)=x, x(0)=y\right\} .
\end{aligned}
$$

In order to solve completely Eq. (8) one needs to make use of the Hamilton equations $\dot{x}(s)=\partial H / \partial p$ and $\dot{p}(s)=$ $-\partial H / \partial x$ together with the boundary conditions $x(t)=x$, $x(0)=y$.

\section{EXPONENTIAL INITIAL CONDITIONS}

One of the most studied initial conditions is the decaying exponential

$$
g(x)= \begin{cases}1, & x<0 \\ e^{-\alpha x}, & x>0\end{cases}
$$

We study in this section how this initial condition affects the speed of the front for (1) parabolic reaction diffusion, (2) heterogeneous parabolic reaction-diffusion, and (3) hyperbolic reaction diffusion.

\section{A. Parabolic reaction diffusion}

Let us study the Fisher-KPP equation,

$$
\partial_{t} \rho=\partial_{x x} \rho+\rho(1-\rho),
$$

which under the condition (9) has been widely studied $[9,11,17]$. However, we want to illustrate how the method presented here yields to the same result. The HamiltonJacobi equation corresponding to Eq. (10) is $\partial_{t} G+\left(\partial_{x} G\right)^{2}$ $+1=0$ and $H=p^{2}+1$. From Eq. (8) one obtains

$$
\begin{gathered}
G(x, t)=\min _{y \geqslant 0}\left[\alpha y+\frac{(x-y)^{2}}{4 t}-t\right], \\
G(x, t)=\min _{y \leqslant 0}\left[\frac{(x-y)^{2}}{4 t}-t\right] .
\end{gathered}
$$

The minimum value in Eq. (11) is attained for $y^{*}=x-2 \alpha t$ $\geqslant 0$ and $G(x, t)=\alpha x-t-\alpha^{2} t$, which holds only for $x$ $\geqslant 2 \alpha t$. The position of the front is then $x(t)=\left(\alpha+\alpha^{-1}\right) t$, which together with $x \geqslant 2 \alpha t$ yields the speed $v=\alpha+\alpha^{-1}$ 
under the restriction $\alpha<1$. When the equality in $x \geqslant 2 \alpha t$ holds one observes that the minimum is attained for $y^{*}=0$ and from Eq. (11) or Eq. (12) $G(x, t)=\left(x^{2} / 4 t\right)-t$, the position of the front is given by $x(t)=2 t$, the speed is 2 and $\alpha$ $=1$. On the other hand, the minimum value in Eq. (12) is attained also for $y^{*}=0$ and the speed is also 2. Therefore, one concludes that

$$
v= \begin{cases}\alpha+\frac{1}{\alpha}, & \alpha<\alpha^{*} \\ 2, & \alpha \geqslant \alpha^{*},\end{cases}
$$

where $\alpha^{*}=1$. It is interesting to note that the speed is a continuous function of $\alpha$ since $\alpha+\alpha^{-1}$ reaches the minimum value just for $\alpha=1$.

We have also explored the case of a Gaussian initial condition, that is,

$$
h(x)=\alpha x^{2}
$$

in Eq. (7). This initial condition has been used in analyzing the growth of bacterial colonies [8]. Exact analytical results have also been obtained. From Eq. (8),

$$
G(x, t)=\min _{y \geq 0}\left[\alpha y^{2}+\frac{(x-y)^{2}}{4 t}-t\right]
$$

and for $y \leqslant 0$ we have Eq. (12). The minimum in Eq. (14) is attained for $y^{*}=x(1+4 \alpha t)^{-1}$, which is always positive and therefore

$$
G(x, t)=\frac{\alpha x^{2}-4 \alpha t^{2}-t}{1+4 \alpha t},
$$

where the position of the front is $x(t)=[t(1+4 \alpha t) / \alpha]^{1 / 2}$. Finally, the speed of the front is

$$
v(t)=\frac{1}{2 \sqrt{\alpha t}} \frac{1+8 \alpha t^{t \rightarrow \infty}}{\sqrt{1+4 \alpha t}} \rightarrow 2 .
$$

This means that the speed for any $\alpha$ is always equal to 2 , which has also been checked by numerical simulations. The case $h(x)=\alpha x^{3}$ also yields the same result: the asymptotic speed is again $v=2$. This may be understood intuitively because the initial condition with $h(x)=\alpha x^{n}$ with $n>1$ is steeper than for $n=1$, and therefore is closer to the step initial condition.

\section{B. Heterogeneous parabolic reaction diffusion}

We consider now a Fisher-KPP equation, where the heterogeneity is present due to the dependence of the reaction rate on the spatial coordinate. This is important in ecological applications, because the reproduction rate of many species varies with latitude, and this will, in turn, affect the speed of their range expansions [18]. To make the analysis as mathematically simple as possible, we consider a linear dependence on the spatial scale (this is also interesting in practice, because it corresponds to a weakly inhomogenous habitat). Therefore, we start with the problem

$$
\partial_{t} \rho=\partial_{x x} \rho+(1+\varepsilon x) \rho(1-\rho),
$$

with the initial condition (9). From the Hamilton equations and taking into account the boundary conditions $x(t)=x$, $x(0)=y$ we find $x(s)=y+(x-y) / t s+t s-s^{2}$ and

$$
\begin{gathered}
G(x, t)=\min _{y \geqslant 0}\left[\alpha y+\frac{(x-y)^{2}}{4 t}-t-\frac{1}{2} t(y+x)-\frac{1}{12} t^{3}\right], \\
G(x, t)=\min _{y \leqslant 0}\left[\frac{(x-y)^{2}}{4 t}-t-\frac{1}{2} t(y+x)-\frac{1}{12} t^{3}\right] .
\end{gathered}
$$

The minimum value in Eq. (16) is attained for $y^{*}=x-2 \alpha t$ $+t \geqslant 0$ and $G(x, t)=\alpha x-t-\alpha^{2} t+\alpha t^{2}-x t-\frac{1}{3} t^{3}$ which holds for $x \geqslant 2 \alpha t-t$. The position of the front is

$$
x(t)=\alpha t+\frac{t+\frac{t^{3}}{3}}{\alpha-t},
$$

which together with $x \geqslant 2 \alpha t-t$ implies $t<\alpha<t$ $+\sqrt{1+t^{2} / 3}$. When $x=2 \alpha t-t$ the minimum is attained for $y^{*}=0$ and $G(x, t)=x^{2} / 4 t-t-\frac{1}{2} t x-\frac{1}{12} t^{3}$ so that the position of the front is now given by

$$
x(t)=t^{2}+\frac{2 t\left(3+t^{2}\right)}{\sqrt{9+3 t^{2}}} .
$$

From the minimum value in Eq. (17), one has that the position of the front is given again by Eq. (19). Finally, we can conclude that

$$
v(t)= \begin{cases}\alpha+\frac{1+\varepsilon^{2} t^{2}}{\alpha-t \varepsilon}+\frac{\varepsilon t\left(1+\frac{1}{3} \varepsilon^{2} t^{2}\right)}{(\alpha-\varepsilon t)^{2}}, & \varepsilon t<\alpha<\varepsilon t+\sqrt{1+\frac{\varepsilon^{2} t^{2}}{3}} \\ 2 t \varepsilon+\frac{2\left(2 \varepsilon^{2} t^{2}+3\right)}{\sqrt{9+3 \varepsilon^{2} t^{2}}} & \text { otherwise, }\end{cases}
$$




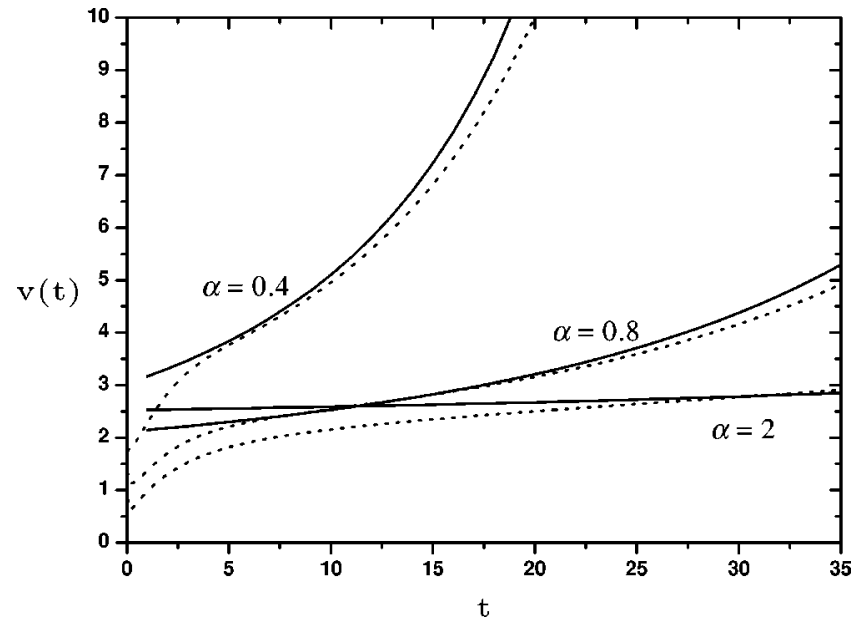

FIG. 1. Comparison between analytical (solid curves) speeds given by Eq. (20) and those from numerical integrations of the partial differential equation (dashed curves), for the temporal evolution of the speed of front in heterogeneous PRD given in Eq. (15). We have taken $\varepsilon=0.01$ and $e^{-\alpha x}$ for $x \rightarrow \infty$. We have employed dimensionless variables.

once the hyperbolic scaling is inverted. In Fig. 1 we show the comparison between Eq. (20) and the speed of fronts obtained from numerical integrations of Eq. (15) together with Eq. (9). We observe that, for several values of $\varepsilon$, there is a good agreement. For some initial conditions, the speed of the front increases with time, but for the initial condition in Eq. (9) with large values of $\alpha$, that is, for steep enough initial conditions, the growth is damped by the effect of the initial condition.

\section{Hyperbolic reaction diffusion}

(HRD) equations have been recently applied in biophysics to model the spread of humans [19], viruses [20], forest fires [21], in population dynamics [22] and combustion flames [23]. The main property of these equations is that they incorporate a delay or memory which is important in many applications. However, there are two kind of such equations frequently used in literature. In general, both may be written under the form

$$
a \partial_{t t} \rho+\left[1-\gamma f^{\prime}(\rho)\right] \partial_{t} \rho=\partial_{x x} \rho+f(\rho),
$$

where $f^{\prime}(\rho)$ stands for $d f / d \rho$ and the parameter $\gamma$ may be equal to $a[19,21-23]$ or 0 [24-26]. The Hamilton-Jacobi associated with Eq. (21) is $a\left(\partial_{t} G\right)^{2}-(1-\gamma) \partial_{t} G-\left(\partial_{x} G\right)^{2}$ $-1=0$ and the Hamiltonian is

$$
H=-\frac{1-\gamma}{2 a}+\frac{1}{2 a} \sqrt{(1-\gamma)^{2}+4 a\left(1+p^{2}\right)} .
$$

From the Hamilton equations we find $x(s)=[(x-y) / t s$ $+y]$ and from Eq. (8) we have

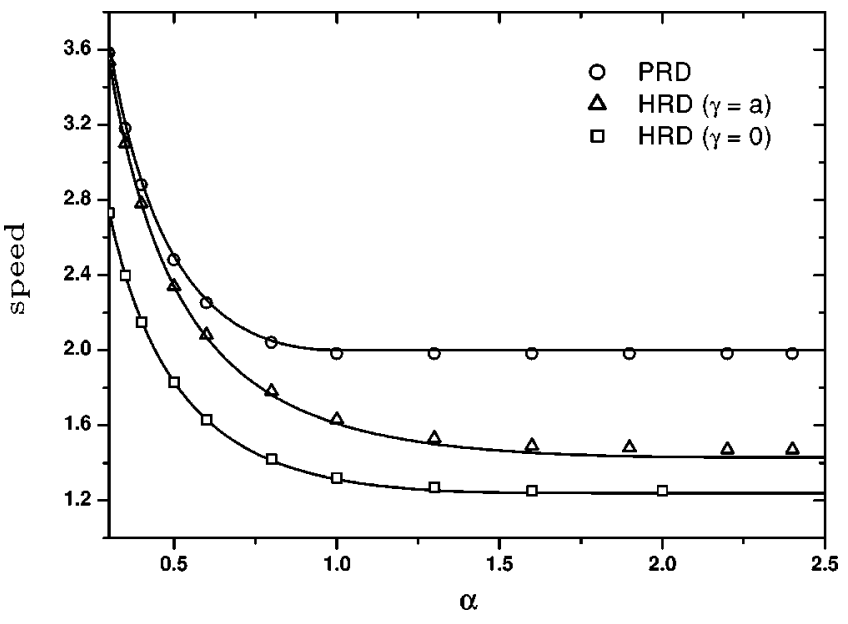

FIG. 2. Comparison between analytical (solid curves) solution for the speed of fronts in HRD given by Eq. (25) and the results of numerical simulations (symbols) together with the PRD case ( $a$ $=0)$. For HRD equations we took $a=0.4$, so that $\alpha^{*}=2.33$ for $\gamma=a$ and $\alpha^{*}=1.61$ for $\gamma=0$. In this plot, the initial condition is given by Eq. (9) and we have employed dimensionless variables.

$$
\begin{gathered}
G(x, t)=\min _{y \geqslant 0}\left[\alpha y+\frac{1-\gamma}{2 a} t\right. \\
\left.-\frac{\sqrt{(1-\gamma)^{2}+4 a}}{2 a} t \sqrt{1-a\left(\frac{x-y}{t}\right)^{2}}\right], \\
G(x, t)=\min _{y \leqslant 0}\left[\frac{1-a}{2 a} t-\frac{\sqrt{(1-\gamma)^{2}+4 a}}{2 a} t \sqrt{1-a\left(\frac{x-y}{t}\right)^{2}}\right] .
\end{gathered}
$$

From the first equation in Eq. (22) we find that the minimum is attained for $y^{*}=x-2 t \alpha\left[(1-\gamma)^{2}+4 a\left(1+\alpha^{2}\right)\right]^{-1 / 2}$ and

$$
G(x, t)=\alpha x+\frac{1-\gamma}{2 a} t-\frac{t}{2 a}\left[(1-\gamma)^{2}+4 a\left(1+\alpha^{2}\right)\right]^{1 / 2},
$$

which holds only for $x \geqslant 2 t \alpha\left[(1-\gamma)^{2}+4 a\left(1+\alpha^{2}\right)\right]^{-1 / 2}$. This condition, together with the position of the front coming from Eq. (23), is equivalent to $\alpha \leqslant \alpha^{*}$, where

$$
\alpha^{*}=\frac{\sqrt{(1-\gamma)^{2}+4 a}}{1-\gamma}
$$

which needs $\gamma<1$ in order to guarantee the positivity of $\alpha$. For $x \leqslant 2 t \alpha\left[(1-\gamma)^{2}+4 a\left(1+\alpha^{2}\right)\right]^{-1 / 2}$ the minimum is attained for $y^{*}=0$ and

$$
G(x, t)=\frac{1-a}{2 a} t-\frac{1+a}{2 a} t \sqrt{1-a\left(\frac{x}{t}\right)^{2}} .
$$

Finally, one has 


$$
v= \begin{cases}\frac{1}{2 a \alpha}\left[\sqrt{(1-\gamma)^{2}+4 a\left(1+\alpha^{2}\right)}-1+\gamma\right], & \alpha \leqslant \alpha^{*} \\ \frac{2}{\sqrt{(1-\gamma)^{2}+4 a}}, & \alpha \geqslant \alpha^{*} .\end{cases}
$$

We observe that $v$ is also a continuous function of $\alpha$ where it depends explicitly on $a$, which represents the quotient between the delay time and the reproduction characteristic time [27].

In Fig. 2 we plot Eqs. (13) and (25) for the cases $\gamma$ $=0, a$ together with the numerical simulations. The effect of the delay is clear: it reduces the speed of propagation relative to the parabolic case. The first part of the result given in Eq. (25) has been recently derived in Ref. [28] by using the method proposed by Mollison [9].

\section{FRONTLIKE INITIAL CONDITION}

Initial conditions different from that studied above may also be considered in the Hamilton-Jacobi formulation, as we show in this and in the following sections. We propose now to study the frontlike initial condition

$$
g(x)= \begin{cases}1, & x<0 \\ \left(\frac{2}{1+e^{\alpha x}}\right)^{b}, & x>0\end{cases}
$$

with $\alpha$ and $b$ positive definite constants for parabolic reaction-diffusion equation (10). In this case it is easy to identify $h(x / \varepsilon)=s \ln \left(1+e^{\alpha y / \varepsilon}\right)$ and from Eq. (8) we have

$$
\begin{gathered}
G(x, t)=\min _{y \geqslant 0}\left[b \ln \left(\frac{1+e^{\alpha y}}{2}\right)+\frac{(x-y)^{2}}{4 t}-t\right], \\
G(x, t)=\min _{y \leqslant 0}\left[\frac{(x-y)^{2}}{4 t}-t\right],
\end{gathered}
$$

once the hyperbolic scaling is inverted. For the first of the equations in Eq. (27) the minimum is attained for $y^{*}>0$ such that it fulfills the following transcendent equation

$$
\alpha b \frac{e^{\alpha y^{*}}}{1+e^{\alpha y^{*}}}=\frac{x-y^{*}}{2 t} .
$$

Equation (28) has one and only one solution $y^{*}$ positive defined provided that $x \geqslant \alpha b t$. For $x \geqslant \alpha b t$ the minimum is attained for $y^{*}=0$ as one can see from both equations in Eq. (27) and the position of the front is the classical $x(t)=2 t$ which holds for $\alpha \leqslant \alpha^{*}$. Whence

$$
\begin{gathered}
G(x, t)=b \ln \left(\frac{1+e^{\alpha y^{*}}}{2}\right)+t b^{2} \alpha^{2} \frac{e^{2 \alpha y^{*}}}{\left(1+e^{\alpha y^{*}}\right)^{2}}-t \text { for } \alpha \leqslant \alpha^{*}, \\
G(x, t)=\frac{x^{2}}{4 t}-t \text { for } \alpha \geqslant \alpha^{*}
\end{gathered}
$$

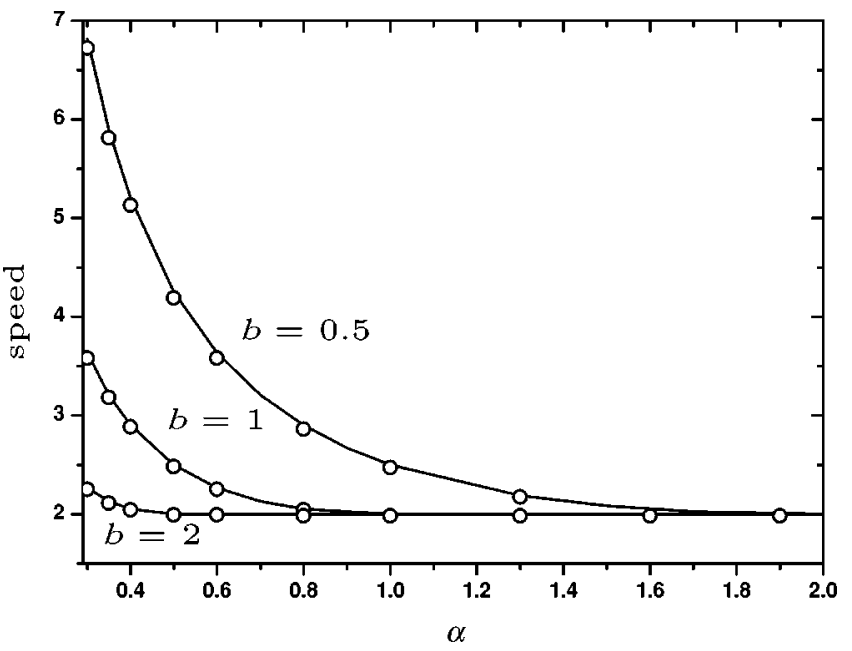

FIG. 3. Comparison of the speed of fronts for PRD equations with the frontlike initial condition given in Eq. (26). Circles are numerical solutions and solid curves correspond to the solution of Eq. (29). We have computed the speed for $b=\frac{1}{2}, 1,2$ and we have observed that for $b$ large the speed is closer to 2 . This is due to the fact that the initial condition is steeper, the higher the value of $b$ is. We have employed dimensionless variables.

where $y^{*}$ is the solution of Eq. (28) and $\alpha^{*}=2 / b$. In order to compute the position of the front and the speed for $\alpha \leqslant 2 / b$ we must solve numerically $G(x, t)=0$ for the first of the equations in Eq. (29) obtaining $y^{*}$ and substituting into Eq. (28) to find the corresponding $x(t)$. The speed may be calculated easily from the slope of $x(t)$ versus $t$. For $\alpha \geqslant 2 / b$ the speed is 2. Fig. 3 displays this semianalytic result, as well as that obtained from numerical simulations of Eq. (10) together with Eq. (26). There is a very good agreement.

\section{ALGEBRAIC INITIAL CONDITIONS}

In this case the technical difficulties to solve the problem analytically are the same as before. Algebraic initial conditions may be represented in a general form as

$$
g(x)= \begin{cases}1, & x<0 \\ \frac{1}{(1+x)^{\alpha}}, & x>0 .\end{cases}
$$

From Eq. (8)

$$
\begin{gathered}
G(x, t)=\min _{y \geqslant 0}\left[\alpha \ln (1+y)+\frac{(x-y)^{2}}{4 t}-t\right], \\
G(x, t)=\min _{y \leqslant 0}\left[\frac{(x-y)^{2}}{4 t}-t\right] .
\end{gathered}
$$

The minimum value for the function in brackets for the first of equations (31) is reached for $y^{*}=0$ or $y^{*}=[x-1$ $\left.+\sqrt{(x+1)^{2}-8 \alpha t}\right] / 2$. The position of the front must be calculated by replacing $y$ in the first equation of Eq. (31) by $\left[x-1+\sqrt{(x+1)^{2}-8 \alpha t}\right] / 2$. From the second one it is 
reached only for $y^{*}=0$ and the position of the front is $x(t)$ $=2 t$. We have checked the position of the front for any time for different values of $\alpha$. We have observed that the slope is for large times independently of $\alpha$ and it is equal to 2. This may be also understood from simple qualitative arguments. If we expect fronts traveling with constant speed for large times then the position of the front grows like $t$ and $y^{*}$, if it is different from 0 , grows also like $t$. In consequence, $\ln (1$ $\left.+y^{*}\right) \sim \ln (t)$ and from the first equation in Eq. (31) $G(x, t)$ $\sim\left(x-y^{*}\right)^{2} / 4 t-t$. Taking $G(x, t)=0$ one has $v=2$. Whence one can conclude that for any value of $\alpha$ the speed of front starting from Eq. (30) reaches a speed equal to 2 for sufficiently large times. This argument is supported also by numerical simulations, which we have performed directly from Eqs. (10) and (30).

\section{CONCLUSIONS}

We have made use of Hamilton-Jacobi theory to study the effect of the initial conditions on the speed of reactiondiffusion fronts with KPP kinetics. The Hamilton-Jacobi framework has allowed us to investigate the transition between quenched and nonquenched fronts both for PRD and HRD equations. Contrarily to the method by Mollinson $[9,11]$, our approach has made it possible to solve the prob- lem for algebraic and frontlike initial conditions. This extends the results by Aronson and Weinberger. We have also dealt with the effect of the initial conditions on the speed of front in PRD equations when spatial heterogeneities are present. We have found agreement between analytical and numerical calculations for all the situations studied. Our work shows that also nonexponential initial profiles affect the speed of fronts.

There remain some interesting open questions. On the theoretical side, it would be interesting to see the effect of a cutoff in the reaction rate, which has been proposed in order to take into account the discretness of reaction-diffusion systems at the microscopic scale $[29,30]$ and may be important in the study of bacterial colonies [31]. On the experimental side, our work may be useful in bacterial colonies [8] and the spread of virus infections [20] since, in both cases, the concentration profile may be measured experimentally.

\section{ACKNOWLEDGMENTS}

Computing equipment used was funded in part by the Generalitat de Catalunya under Grant No. SGR-2001-00186 (V.M. and J.F.), and by the Ministry of Science and Technology under Grant Nos. BFM 2000-0351 (V.M. and J.F.) and REN 2000-1621 CLI (J.F. and T.P.).
[1] R.A. Fisher, Ann. Eugenics 7, 35 (1937).

[2] A.N. Kolmogorov, I.G. Petrovskii, and N.S. Piskunov, Bull. Mosc. Univ. Math. Ser. A 1, 1 (1937).

[3] D.G. Aronson and H.F. Weinberger, in Partial Differential Equations and Related Topics, edited by J.A. Goldstein, Lecture Notes in Mathematics Vol. 446 (Springer, Berlin, 1975), pp. 5-49.

[4] D.G. Aronson and H.F. Weinberger, Adv. Math. 30, 33 (1978).

[5] H.F. Weinberger, in Nonlinear Partial Differential Equations and Their Applications, edited by J. Chadam (Springer-Verlag, New York, 1978), pp. 47-96.

[6] H.F. Weinberger, SIAM (Soc. Ind. Appl. Math.) J. Math. Anal. 13, 353 (1982).

[7] J. Fort and V. Méndez, Rep. Prog. Phys. 65, 895 (2002).

[8] M.J. Grimson and G.C. Barker, Phys. Rev. E 49, 1680 (1994).

[9] D. Mollison, J. R. Stat. Soc. Ser. B. Methodol. 39, 283 (1977).

[10] M. Ablowitz and A. Zeppetella, Bull. Math. Biol. 41, 835 (1979).

[11] J.D. Murray, Mathematical Biology (Springer-Verlag, Berlin, 1989).

[12] S. Puri, Phys. Rev. A 43, 7031 (1991); S. Puri and A.J. Bray, J. Phys. A 27, 453 (1994).

[13] D.J. Needham and A.N. Barnes, Nonlinearity 12, 41 (1999).

[14] S. Fedotov, Phys. Rev. E 59, 5040 (1999); 58, 5143 (1998).

[15] M. Freidlin, Functional Integration and Partial Differential Equations (Princeton University Press, Princeton, NJ, 1985), Sec. 6.3.
[16] M. Freidlin, in Semi-linear PDE's and Limit Theorems for Large Deviations, edited by P.L. Hennequin, Lectures Notes in Mathematics 1527 (Springer, Berlin, 1992), pp. 1-109.

[17] D.J. Needham, Q. J. Mech. Appl. Math. 45, 469 (1992); J.A. Sherratt, Dyn. Stab. Syst. 13, 167 (1998).

[18] D.A. Andow, P.M. Kareiva, S.A. Levin, and A. Okubo, in Evolution of Insect Pests: the Pattern of Invasions edited by K. C. Kim (Wiley, New York, 1993).

[19] J. Fort and V. Méndez, Phys. Rev. Lett. 82, 867 (1999).

[20] J. Fort and V. Méndez, Phys. Rev. Lett. 89, 178101 (2002).

[21] V. Méndez and J.E. Llebot, Phys. Rev. E 56, 6557 (1997).

[22] V. Méndez and J. Camacho, Phys. Rev. E 55, 6476 (1997).

[23] J. Fort, T. Pujol, and A.S. Cukrowski, J. Phys. A 33, 6953 (2000).

[24] J.M. Sancho and A. Sánchez, Phys. Rev. E 63, 056608 (2001).

[25] V. Méndez, T. Pujol, and J. Fort, Phys. Rev. E 65, 041109 (2002).

[26] K.K. Manne, A.J. Hurd, and V.M. Kenkre, Phys. Rev. E 61, 4177 (2000).

[27] V. Méndez, J. Fort, and J. Farjas, Phys. Rev. E 60, 5231 (1999).

[28] G. Abramson, A.R. Bishop, and V.M. Kenkre, Phys. Rev. E 64, 066615 (2001).

[29] E. Brunet and B. Derrida, Phys. Rev. E 56, 2597 (1997).

[30] D.A. Kessler and H. Levine, Nature (London) 394, 556 (1998).

[31] E. Ben-Jacob, I. Cohen, and H. Levine, Adv. Phys. 49, 395 (2000) 